the exception of the formation of a secondary abscess in the right shoulder, which much disabled him for a time. A sinus remained in the stump, with two or three prominent granulations, which indicated the presence of necrosis of a portion of the shaft of the femur; this was associated with a constant dull pain. On the 8th of October, Mr. Fergusson cut down upon the end of the stump, and found that the bone was in a state of necrosis, but the fragment was firmly attached; and by the aid of a strong pair of forceps, and by the use of considerable force, it was detached and pulled out, and found to be a fragment fully six inches long, occupying one half of the circumference of the bone. This, although contrary to the expectations of everyone present, was, to some extent, explained on remembering that at the time of the amputation the upper portion of bone removed above the seat of fracture was but three inches long. It is not improbable that the vitality of the shaft was impaired by the non-union of the fracture; and hence the death of so much of it. It is now confidently hoped that a permanent recovery may ensue free from the many drawbacks which have presented themselves during the long period of illness under which this patient has laboured.

On the same occasion as the removal of this piece of dead bone, another example of ununited fracture was submitted to operative measures. A young man, also a sailor, (and here we would remark, that the majority of such cases occur in seafaring men,) hopped into the theatre on one leg, and leaped upon the operating table with considerable agility. His injury was a simple fracture of the centre of the shaft of the left tibia six months before, but which had resisted union, and now formed a false joint. Mr. Fergusson scraped the ends of the bones with a tenotomy knife, and introduced a wire seton between them, which may prove serviceable, as other things seem favourable for union in this instance.

The necrosis of the end of the stump, in Mr. Fergusson's patient, has reminded us of a young soldier who had his left arm taken off by primary amputation at the siege of Lucknow, and who was a patient in St. George's Hospital this time last year with necrosis of the end of the bone. There was a good deal of tenderness remaining in the stump. On the 14th of October, 1858, when the influence of chloroform was fully manifested, Mr. Pollock reopened the old cicatrix, and removed the portion of necrosed bone, with, as we afterwards learnt, very good results.

\section{STONE IN THE BLADDER OF A CHILD, REMOVED BY ALLARTON'S MEDIAN OPERATION.}

\section{(Under the care of Mr. Bowman.)}

In the vast majority of cases the ordinary well-known lateral operation for the removal of stone in children is attended with success. Indeed, after seeing it performed, and watching the results, in we might say upwards of a hundred cases in the young, a fatal or untoward termination is a circumstance com. paratively rare. Difficulties, however, which are familiar to most surgeons, sometimes arise in its performance. The principal of these is the recession before the finger of the neck of the bladder, from the readiness with which the parts yield in children in comparison with adults.

On Saturday, the 8th instant, the pupils of this hospital had the opportunity of seeing Allarton's operation performed by Mr. Bowman upon a child, who had been labouring under symptoms of stone for some time. It is described as a modifi. cation of the Marian or apparatus major operation, now looked upon as somewhat ancient, and consists, after the patient has been placed in the usual position with a deep-grooved staff in his bladder, of pushing a long sharp-pointed bistoury, with its edge turned upwards, into the middle line of the perinæum, a little above the anal aperture, so as to open the membranous portion of the urethra immediately in front of the apex of the prostate. The finger in the rectum serves as a guide to this part of the canal, and prevents the point of the knife from wounding the gut. On reaching the staff, the incision is continued upwards to the required extent. A steel ball-probe is now passed along the groove into the bladder, and the staff is withdrawn. This probe has now to serve the surgeon as a guide, by means of which he passes his left index-finger into the bladder, gradually dilating the prostatic portion of the urethra and the neck of the bladder as he proceeds, and when this is accomplished he feels the stone, withdraws the probe, and extracts the calculus with a pair of forceps in the usual way.

On the few occasions at which we have been present, the operation was done in the manner described, and the same steps were taken by Mr. Bowman. He, however, "encountered great difficulty," as he expressed himself, " in getting the finger in to the bladder through the wound in the urethra. It passed above, below, on either side, and the tendency was not to get in through the urethra. If this operation is to be performed, there must be some means devised of dilating the urethra before passing the finger into the bladder."

After the lapse of some twenty minutes or half an hour, $\mathrm{Mr}$. Bowman finally succeeded in extracting a pretty large calculus, and the operation was ended. The operator hoped that, notwithstanding the apparent violence and protracted nature of the procedure, the child would do well. He, however, stated that he had no reason to be dissatisfied with the lateral operation in children, but resorted to the median on the present occasion so as to give those present an opportunity of witnessing it. A week afterwards we found the little patient going on extremely well.

Amongst other advantages insisted on by the advocates of this operation is, the absence of hæmorrhage. Now, in all the cases in which we have seen it performed, there certainly did not appear to be less bleeding than in the usual lateral method; and in one instance, under Mr. Cock's care at Guy's, we recollect that there was a great deal of bleeding, which much weakened the patient.

Since writing the foregoing, we have again seen the median operation resorted to at Guy's Hospital. On the 10th instant Mr. Cock operated upon a boy between eight and nine years of age, who had had a very large stone in his bladder for some years. We observed that the use of the probe was dispensed with, as the finger readily entered the bladder before the staff was withdrawn; and it was found necessary to cut right into the rectum through the sphincter, to permit of the ready extraction of what proved to be one of the largest calculi ever removed from a child. It was got away in two portions.; one formed an accurate cast of the bladder itself, whilst the other was attached to it like the spout of a teapot, and had evidently occupied the prostatic portion of the urethra, which had become considerably dilated.

\section{MTDDLESEX HOSPITAL.}

EFFCTS OF A HORSE-BITE.

(Under the care of Mr. Flower.)

THE wounds produced by the bites of animals often take on a most unhealthy action, and, besides destroying the parta which have been injured, occasionally prove fatal; and this even when the animal is healthy. An elderly and stout man was recently admitted into the Middlesex Hospital, under Mr. Flower's care, suffering from the effects of a bite on his right hand by a horse. The part more particularly injured was the forefinger, which was much lacerated and bruised, and formed a most unhealthy wound, accompanied by suppuration, with finally sloughing of some of the tendons of the long flexors. It was impossible, therefore, to obtain a useful finger in the event of the morbid action being arrested, and accordingly the entire digit was removed by Mr. Flower, including the metacarpo-phalangeal joint. There was much sanguineous oozing, and the wound was not fairly closed until two or three hours afterwards, which was accomplished by means of metallic (iron wire) sutures. The man is now doing well.

\section{FORCIBLE FLEXION OF ANCHYLOSED KNEES.}

(Under the care of Mr. Mitchell HenRy.)

The forcible rupture of the uniting medium between the apposed surfaces of the knee, after the cessation of all active disease, we saw put into practice, at the Westminster Hospital, on the 4 th instant, by Mr. Holthouse, and at the Middlesex Hospital, on the 5th instant, by Mr. Mitchell Henry.

Mr. Holthouse's patient is a girl of nineteen years, who suffered from disease of the knee-joint, which got quite well, but was followed by shortening and contraction of the muscles at the back of the joint, with partial flexion of the knee, and consequent lameness. It became expedient to divide the ham. string muscles here, and this was at once followed by some straightening spontaneously, the complete restoration to natural position being afterwards gradually accomplished by means of proper instruments.

At the Middlesex Hospital, the patient was a girl aged twenty-two, who suffered from disease of the knee for seven 